\title{
Extraction of subjective properties in image processing
}

\author{
J.A. Martin-Pereda ${ }^{*}$, A. González-Marcos \\ E.T.S. Ingenieros de Telecomunicación. Universidad Politécnica de Madrid
}

\begin{abstract}
Most of the present digital images processing methods are related with objective characterization of external properties as shape, form or colour. This information concerns objective characteristics of different bodies and is applied to extract details to perform several different tasks. But in some occasions, some other type of information is needed. This is the case when the image processing system is going to be applied to some operation related with living bodies. In this case, some other type of object information may be useful. As a matter of fact, it may give additional knowledge about its subjective properties. Some of these properties are object symmetry, parallelism between lines and the feeling of size. These types of properties concerns more to internal sensations of living beings when they are related with their environment than to the objective information obtained by artificial systems.

This paper presents an elemental system able to detect some of the above-mentioned parameters. A first mathematical model to analyze these situations is reported. This theoretical model will give the possibility to implement a simple working system. The basis of this system is the use of optical logic cells, previously employed in optical computing.
\end{abstract}

Keywords: Illusion, perception, size perception

\section{INTRODUCTION}

To extract information about object shapes or dimensions is the main task for every image processor working nowadays. There is a clear reason to perform this type of processing. Information obtained is employed in many different applications ranging from objects classification to pattern detection. Any one of these applications concerns objective properties and results are employed in a particular situation or compared with other similar results. The final objective is, for example, to detect if two fingerprints are the same or if the signature of a certain plane corresponds to a friendly aircraft or to an enemy. These situations need to obtain absolute results with real data. But there is other group of situations where the obtained results should be in a certain type of agreement with the particular impression obtained by human beings. Many examples may be given. Some are related, for instance, to the structures given by architects to their buildings. It is a well-known fact that some of the Greek temples have their columns not exactly perpendicular to their basements. They adopt a certain angle, different to $90^{\circ}$, in order to give the observer the impression of being perpendicular. Location and shapes change slightly the felling had by the observer with respect to real properties. A more common situation refers to the stripes and different lines adopted in dresses, clothes or any fashion costume. According to orientation and relation between them, any person wearing them may look thicker o thinner. These results have nothing to do with the objective properties above indicated and with the real data that any conventional image processor could give. The reason refers better to subjective properties of objects, as being perceived by human beings, than to objective properties extracted by mechanical methods.

The analysis of above facts, although the ancient Greeks knew several, has being studied since the middle of the XIX century. The first scientific description in modern times is in a letter from Swiss naturalist L.A. Necker, who wrote in 1832 that a drawing of a transparent rhomboid reverses in depth: sometimes one face appears to be in front and sometimes the other. This effect is illustrated with an isometric cube rather than with Necker's original figure. Moreover, the simplest distortion illusion was also the first to be studied. This is the horizontal-vertical illusion, which was described by W. Wundt. This illusion is simply that a vertical line looks longer than a horizontal line of equal length. But perhaps the most famous of all distortions is the double-headed-arrow figure devised by Franz Müller-Lyer and presented in 15 variations in 1889. This figure is so simple and the distortion is so compelling that it was immediately accepted as a primary target for theory and experiment. The influence of angles is clear with respect to their influence on the perception of length. It may be seen, by

\footnotetext{
* Correspondence: Email jamp@tfo.upm.es; E.T.S. Ingenieros de Telecomunicación. Universidad Politécnica de Madrid, Ciudad Universitaria. 28040 Madrid. Spain.
} 
simple measurements, that the horizontal part has the same length in both figures, but the line on the upper row appears considerably shorter than the other one. This illusion has had many possible explanations and many theories have been advanced. Some authors gave an eye-movement theory. Other groups pointed out that the "wings" of the arrowheads drew attention away from the ends of the central line, thus making it expand or contract. These theories have a clear origin: they are "physiological" theories because they involve disturbance in the information channels rather than inappropriateness of how the signaled information is being applied to the perceptual situation. But, as several authors have pointed it out, this illusion occurs though the usual lines joining the arrows are left out. It even persists if the figure is reduced to three dots for each arrow. Many other theories have appeared in the last years. Some of then are "cognitive" theories, as the "empathy" theory. The idea is that the observer identifies himself with parts of the figure and that he becomes emotionally involved so that his vision is distorted rather as emotion may distort an intellectual judgment. In the arrow illusion, it would be argued that the outgoing arrow suggests, emotionally, expansion which one then sees. But the reality is that the illusion, in this case, gives distortion whatever one's mood, and continues to do so in any different circumstance. The last theory to be indicated here is the "perspective" theory. In this theory, the central idea is that the illusion figures suggest depth by perspective, and that this suggestion of depth produces size changes. The illusion figures can be thought of flat projections of threedimensional spaces and so a simple generalization holds: the parts of illusion figures, which would represent distant features, are enlarged, and those parts corresponding to near features are shrunk. In the Müller-Lyer illusion the arrow with the outgoing fins represent the inside corner of a room and the outgoing arrowheads represent an outside corner of a building. A problem pointed by some detractors of this theory is that how could "suggestions of distance" produce changes of apparent size. Some other interpretations have been studied in the last years, being one of the better known the "Size Constance". This is the tendency for the perceptual system to compensate for changes in the retinal image with changes of viewing distance. The image of an object doubles in size whenever its distance is halved.

The above-indicated theories involve several different interpretations. But in

Fig. 1.- Wundt illusion.

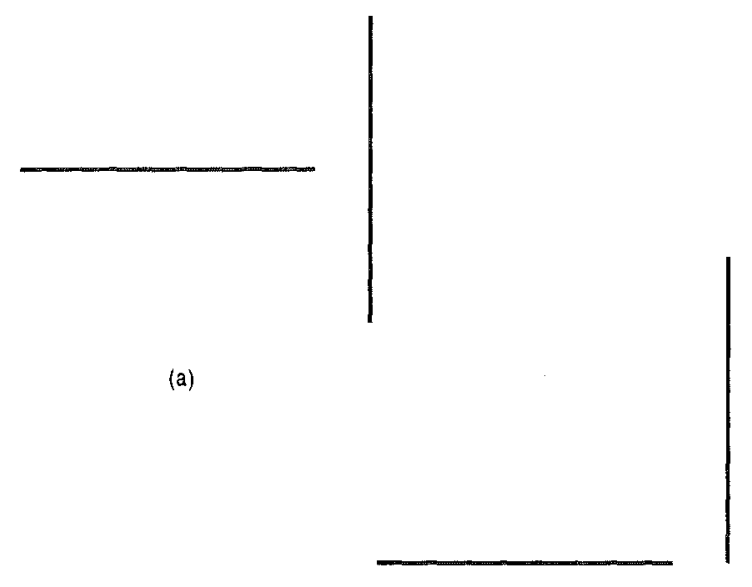

(b)

Fig. 2.- Different approaches to the Wundt illusion. almost everyone there are some common factors. The first one is the importance of angles in determining sizes and parallelism. The other one concerns the importance of the subject looking to some figure. This last aspect is largely related with previous experiences had by the active subject. In the same way that occidental art needed several centuries to reach the concept of perspective in painting, to interpret some images needs a previous education and, as a consequence, some concepts inserted in the observer mind. A certain type of memory acts in every occasion some object appears in the front of the observer. Its interpretation depends on the real image "seen" by the subject and on the latent images internally maintained.

A new theory will be reported in this paper. We will try first to establish some general concepts, most of them based on previous theories, and from them we will develop some rudimentary principles in order to quantify possible illusions. Some of these principles were indicated by in another work and they will get in this paper a more developed form. Moreover, the theory to be reported here is just a first approach to a more general one.

\section{BRIEF GALLERY OF VISUAL ILLUSIONS}

In order to get a close approach to some of the more common illusions, in this paragraph we will show some of the more well known. Some of them will be the basis for the concepts to be developed in the next paragraphs. The review to be presented here is just as an index of different situations and how some changes in their characteristics may affect to the final 
impression had by the observer. A more detailed explanation of their properties may be seen in any of the existing books and papers. Some of them are listed in ${ }^{1-5}$.

As it was pointed out previously, the first reported illusion is the horizontal-vertical illusion, described by W. Wundt, assistant to Helmholtz at Heidelberg. This illusion is simply that a vertical line looks longer than a horizontal line of equal length. But some differences may be seen according to the situation where both lines are located. Fig. 1 shows the case of a vertical line located above the horizontal lines. Both lines have the same length. The illusion, as described by Wundt, is clear: vertical line looks longer than the horizontal one. But illusion is no so clear if the location is different. Fig. 2 shows two different situations. In any of them, both lines are located in similar locations, the only difference being the relative positions. In Fig. 2 (a) they are aligned in the center of the vertical one, in Fig. 2 (b) horizontal line rests at the bottom of the vertical one. If these figures are observed isolated, without any connection between them, some differences may be seen perceived. In Fig. 2 (a) horizontal line no longer looks smaller than the vertical one. In Fig. 2 (b) both lines looks almost with same size. This fact indicates that the relative position of different objects affects to the sensation had by the observer and deserves a further explanation.

The second analyzed effect was the Müller-Lyer illusion. Its main properties have been described in the previous paragraph. But, in the same way than in the Wundt effect we have pointed out some particular situations, we will indicate here some other concerning this effect. These properties are related with the influence of the angles located at the end of (a)

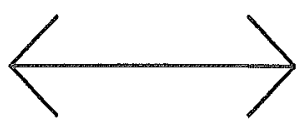

(b)
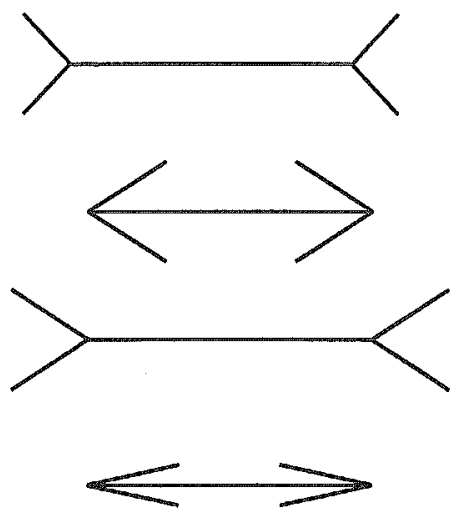

(c)

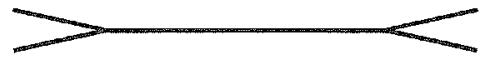

Fig. 3.- Different approaches to the Müller-Lyer illusion.

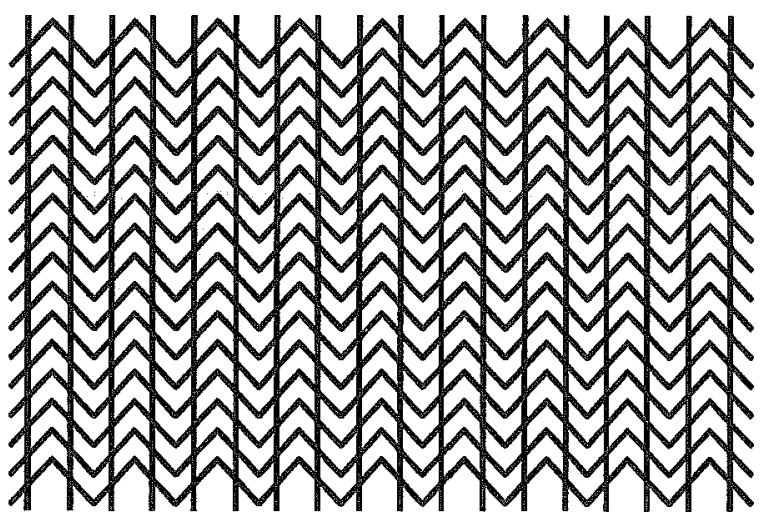

(a)
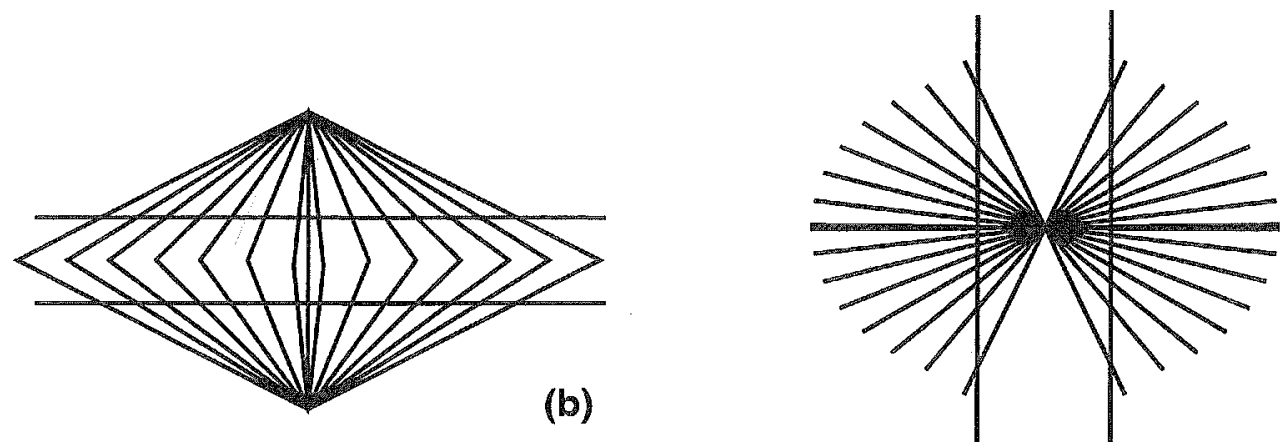

(c)

Fig. 4.- Illusions related with effect on parallelism. (a) Zöllner illusion. (b) Wundt illusion. (c) Hering illusion. 
the line. Three different cases appear in Fig. 3 being the difference these angles. If, as before, the three different conditions are observed isolated, different impression may be seen. Depending on the angle values the difference in perceived length is different. According to some real experiences, the maximum effect corresponds to angles around $45^{\circ}$. This is a clear indication that it is not just the presence of other lines the origin of the effect but their characteristics. The angles at the end of the lines act as a certain "weight" in order to determine the length and this weight depends on the value of these angles. Some of these effects were analyzed by us in a previous paper.

The above-indicated effects may be more clearly seen in the Zöllner's (Fig. 4.a), Wundt's (Fig. 4.b) and Hering's (Fig. 4.c) illusions. Effects are slightly different in every one of
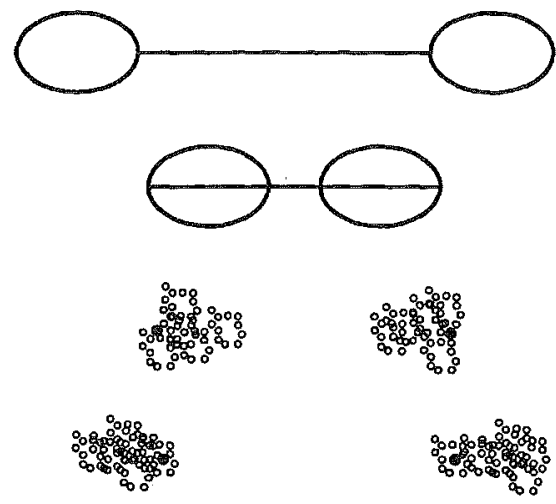

Fig. 5.- Two different approaches to the Müller-Lyer illusion. equality is given by the circles located at their ends. In the second case, it is the distance between the two black dots is the same in both pictures but the separation of the lower pair seems greater than that of the upper pair. This means that there are some other types of effects influencing length than just the presence of angles.

Many other types of illusions may be seen in the literature ${ }^{1-5}$. But the reported here give the principal aspects to be considered in this work in order to extract some facts useful to develop the models we are going to present.

\section{GALLERY OF MAIN FACTS}

Some aspects are needed to be pointed out before we present our simple model to quantify visual illusions. They are related with those facts we consider fundamental to interpret simple sensations, namely those related with parallelism and linear dimension. Two different concepts will be introduced. The first one is related with the feeling of parallelism. In this case, the main operation to be considered is the symmetry operation. Two parallel lines, as in the case of Zöllner, Hering or Wundnt illusions, are characterized for the existence of a symmetry line in between. This line, located at the center, may be considered as a twofold axis. The operation of rotating a figure about this axis is a symmetry operation because carries it to a position impossible to distinguish with respect to the previous one. Moreover, there is one more possible symmetry operation. It is a plane of symmetry perpendicular to the surface that contains the two parallel lines and it crosses the previous symmetry line. Because we are in this case in a two-dimensional situation, we do not consider this plane. These symmetry elements are indicated in Fig. 6.

Together with above indicated symmetry elements there are other aspects to consider. In our present example, symmetry gives a possible operation but together with the parallel lines some other details appear. These details are, in this 
case, the crossing lines forming an angle $\alpha$ with the horizontal segments. These details give a certain "oriented weight" to the segments and, moreover, this weight imposes a new condition to the total operation. As it will be shown later, their presence will be the responsible for the illusion seen.

The second aspect to be considered refers to the characteristics of the place where the object is located. It is clear than when a painted landscape is observed, some characteristics of the scene are appreciated through the presence of a horizon or some other details, as trees, mountains or remote houses. In this case, dimensions are recognized because we compare objects and the position where we guess are located. But when just two lines are in the plane, as in Fig. 1, no references are present. Hence, why the vertical line looks longer than the horizontal line? As we have presented in Fig. 2, this results depends on the relative position of the lines. And this effect is, as it has been clearly proved (see, for instance ${ }^{1}$ ), the result of a previous training or education. In the Western world, people have a visual environment rich in perspective cues to distance. People living in dense forest do not experience distant objects, because they live in small clearances in the forest. Zulus experience the Müler-Lyer arrow illusion to only a small extent, and are hardly affected at all by other distortion illusion figures. A possible reason to the Wundt illusion is the internal representation of a certain horizon. Both lines are internally located in a landscape where the horizon appears somewhere in the figure. According to the vertical and horizontal lines position with respect to it, dimensions are guessed.

According to above indicated facts we have introduced in this paper two fundamental concepts to explain them. The first one is the concept of "visual oriented density" and the second one is the "internal subjective vanishing point", located near the back center of image. These two concepts will allow us to explain some of the illusions mentioned in previous paragraphs.

\section{GALLERY OF NEUROPHYSIOLOGIC FACTS}

This paper is not related with physiological aspects concerning the mammalian visual cortex. But because we will adopt some structural and functional details of that zone, we considerer convenient to remember some of its main aspects.

The most detailed map of the retina is found in area V1 of the mammalian visual cortex. This detail goes close with

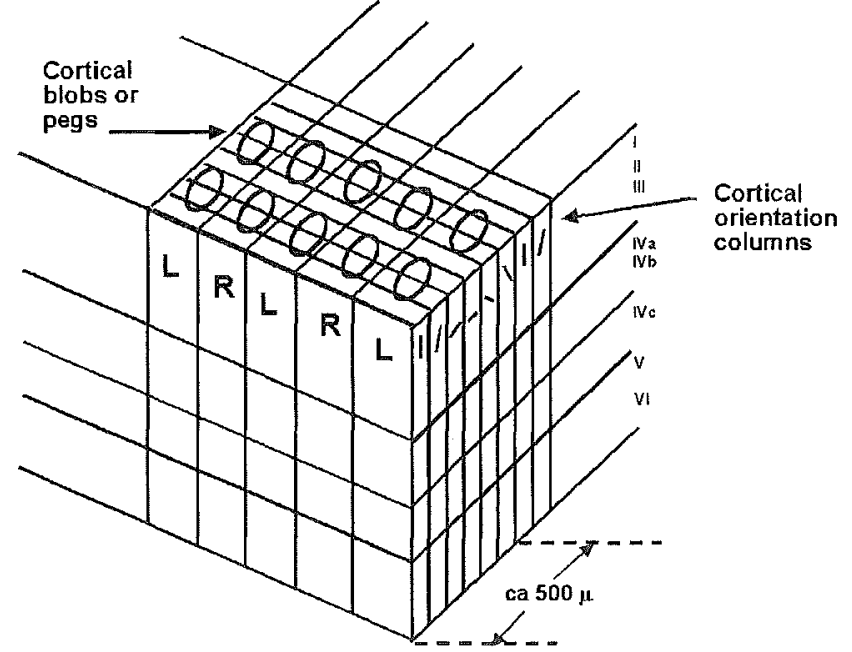

Fig. 7.- Structure of a part of the mammalian visual cortex. a rich functional architecture, uncovered by Hubel and Wiesel in their physiological studies ${ }^{7}$. They proposed that V1 was made up of a set of orientation columns and a set of ocular dominance columns. A way to clarify these ideas is to see how cells encountered in a penetration made perpendicular to the cortical surface of V1 respond.

Some cortical visual neurons have receptive fields with "on" or "off" zones arranged in parallel. Diffuse illumination of the entire receptive field causes little change in the spontaneous activity of them. But if a "bar" of light in the "correct" orientation and position is projected into the receptive field, there is strong activation. These fields are called "simple" receptive fields, because it is easy to establish their functional organization by projecting small spots of light onto different parts of the receptive field. Other type of neurons with "complex" receptive fields covers higher order functions. The whole structure and function of the neuron primary visual cortex architecture is somehow much more complex. Their nerve cells are arranged in six distinct cytoarchitectonic layers parallel to the cortical surface. These nerve cells are organized not only within layers parallel to the surface but also in "columns" perpendicular to it. When the neurons laying at these columns are tested with contours oriented in various directions one finds a progressive change in optimal orientation across adjacent columns. In other words, the cells in a particular column share the same preferred orientation. This orientation changes in a systematic way as one moves across the cortical surface, such that after half a millimetre or so we are back to the first 
orientation. Thus the visual cortex is traversed by a series of bands, within each of which every possible orientation is represented. This fact may be demonstrated anatomically. A highly stylized representation of a slab of visual cortex, showing its organization

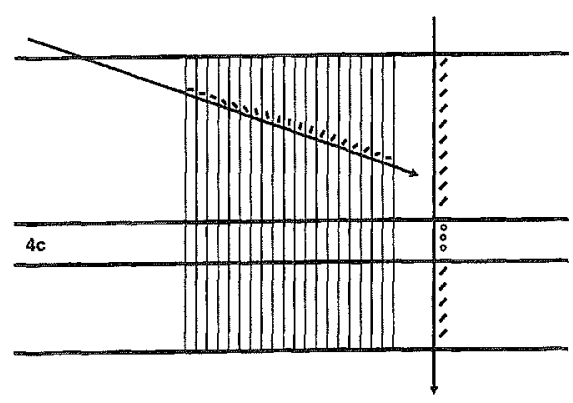

Fig. 8.- Simplified vision of the "icecube" model. into columns, is shown in Fig. 7. It is necessary to add that vision corresponding to right and left eyes are at alternate columns. It is necessary to travel a minimum distance of about $0.8 \mathrm{~mm}$ for the cells to respond to the right eye plus the left eye and then start the sequence again, a distance comprising the orientation hypercolumn. Equally, it is necessary to travel about $1 \mathrm{~mm}$ for all 18 orientations to be covered, a distance comprising the orientation hypercolumn. Each small part of the field of view is screened for one eye and then for the other eye, and each small part is simultaneously screened for different orientations, the entire process being repeated again in the adjacent millimetre for an adjacent small part of the field of view. Colours are detected, in a similar way, at specific places, named cortical blobs o pegs. This structure is sometimes called the "ice-cube" model. A simplified model appears in Fig. 8.

The extraordinary regularity and precision of the retinal map in V1 may have misled to some people into thinking that it must be the retina that is mapped in all visual areas. But the results from studies of the prestriate cortex suggest that it may need a new concept of what a map in the cortex means. Some people are inclined to think of these maps as static maps of the retina. But may be useful to think of them as dynamic maps and even constructional maps. The relation between the external image and image the living body "sees" may have some to do with this aspects of the visual cortex. Without going into more details, we consider that these facts are related in a direct way with the interpretation human beings perform when some subject impinges onto their visual systems. Each direction, in this case, activates a particular column and a nervous current will be processed. If two excited columns are excited and they are close to each other, the total current towards higher sensory systems may be different than just a simple addition of two currents. Some influence between them must be present and hence, a different impression has to be obtained. These facts will be taken into account in the model we propose in the next paragraph. More details about these physiological aspects of the vertebrate visual system may be seen in any physiology textbook. See, for example ${ }^{8-10}$.

\section{ANALYSIS OF THE ZÖLLNER ILLUSION}

Although Zöllner illusion is more related with Fig, 4.a than with Fig 6, we will use the last one because the more important facts, namely symmetry operations, appear in the last one. We will use in this effect the concept of visual oriented density pointed out in a previous paragraph. We define it as the "distributed weight" of a certain detail of the image with respect to the preferential line in the subject. The preferential line is, in this case, the twofold symmetry axis. The distributed weight corresponds to the columns in the physiological model that have been excited in each partial detail of the image seen

by the observer. In the case shown in Fig. 6 just two different

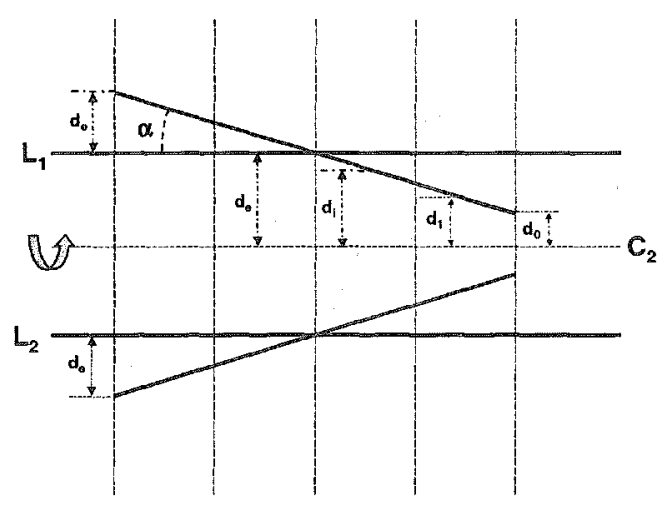

Fig. 9.- Main parameters in the Zöllner illusion. columns will be excited because there just two different line orientations. Some more details are given in Fig. 9. Another point needs now to be considered. Parallelism has imposed the central line as symmetry reference. Hence, the weight of parallel lines must be different to the weight of the crossing lines. According to experimental results, these crossing lines impose a different influence depending on their angle values. Stronger effects correspond to angles between $30^{\circ}$ and $50^{\circ}$. For example, $85^{\circ}$ or $5^{\circ}$ angles do not have almost any effect on parallel illusions.

To analyze this situation, as it has been indicated before, a small part of the total image has been redrawn in Fig. 9. We have divided it in four columns. This indicates our assumption that, in this case, only four cortical orientation columns may be excited. In each one of them, two orientation bands detect two different orientations. We call $2 \mathrm{~d}_{\mathrm{e}}$ the distance between parallel lines, $\alpha$ the angle of the crossing lines with the 
parallel ones and $d_{i}$ the distance from the middle point of each segment in a column to the twofold axis of symmetry. With these data, we propose the following model to calculate the parallelism impression. We establish the following matrix as a main indication to parallelism

$$
\pi_{\beta}=\left[\pi_{\alpha, 1}-\pi_{\alpha, 2}, \pi_{\alpha, 3}-\pi_{\alpha, 4,1, \alpha}\right]
$$

Elements in this matrix are given by

$$
\begin{gathered}
\pi_{\alpha k}=\sigma_{\alpha k} / \delta_{\alpha k} \\
\sigma_{\alpha k}=\left\{\sum_{i=1}^{n} \omega_{i} \delta_{i}\right\}_{\alpha k}^{n}+s\left\{\sum_{j=1}^{n} \omega_{j} \delta_{j}\right\}_{\alpha l}
\end{gathered}
$$

with

where $\omega_{i}$ is the "weight" given to the $\mathrm{i}$-th element and $\delta_{\mathrm{i}}$ its distance to the central line (symmetry axis). $\mathrm{S}$ is the symmetry operation imposed to the object, in our case, the twofold axis. This symmetry operation carries one of the details of the object to the other one. $\sigma_{\alpha \mathrm{k}}$ is a measure of the details that have "impressed" to sensorial columns. $\wp$ is a similar effect corresponding to the parallel lines.

Elements in matrix $\pi$ are zero when parallelism is "seen". The difference with zero gives an idea about how the degree of parallelism is "not seen". A simple example may give a more simple interpretation of this model.

If we assume the following values for above indicated parameters:

$$
\begin{array}{lll}
\delta_{\mathrm{e}}=6 & \delta_{1}=3 & \delta_{\mathrm{i}}=3+2(\mathrm{i}-1)
\end{array}
$$

$\omega=0.5$ every "weight" has the same value in this case, because the considered detail is a segment.

Columns are numbered from right to left.

$$
\wp_{\mathrm{i}}=6 \quad \forall \mathrm{i}
$$

With these data, matrixes corresponding to elements above and below the central line are

$$
\begin{aligned}
\sigma_{1}= & \{6 \times 1+9 \times 0.5 ; 6 \times 1+7 \times 0.5 ; 6 \times 1+5 \times 0.5 ; 6 \times 1+3 \times 0.5\} \\
\sigma_{2}= & \{6 \times 1+9 \times 0.5 ; 6 \times 1+7 \times 0.5 ; 6 \times 1+5 \times 0.5 ; 6 \times 1+3 \times 0.5\} \\
\sigma_{\mathrm{T}}: \quad & \sigma_{1}+\mathrm{C}_{2} \sigma_{2} \quad=\{10.5+10.5 ; 9.5+9.5 ; 8.5+8.5 ; 7.5+7.5\}= \\
= & \{21 ; 19 ; 17 ; 15\} \\
& \wp_{\mathrm{T}}: \quad \wp_{1}+\mathrm{C}_{2} \wp_{2} \quad=\{6+6 ; 6+6 ; 6+6 ; 6+6\}= \\
= & =\{12 ; 12 ; 12 ; 12\} \\
& =\{0.57 ; 0.63 ; 0.70 ; 0.8\}
\end{aligned}
$$

These numbers indicate the existence of a certain "lack of parallelism" in the obtained impression. If we subtract now to the number at a column the corresponding to the adjacent column in the right, we get

$$
\left\{\pi_{\mathrm{T} 1}\right\}=\left\{\pi_{\mathrm{Ti}}-\pi_{\mathrm{T}(\mathrm{i}-1)}\right\}: \quad=\{0.06 ; 0.07 ; 0.1\}
$$

and by successive repeating of this operation

$$
\begin{aligned}
& \left\{\pi_{\mathrm{T} 2}\right\}=\left\{\pi_{\mathrm{T} 11}-\pi_{\mathrm{T} 1(\mathrm{I}-1)}\right\}: \quad=\{0.01 ; 0.03\} \\
& \left\{\pi_{\mathrm{T} 3}\right\}=\left\{\pi_{\mathrm{T} 2 \mathrm{i}}-\pi_{\mathrm{T} 2(\mathrm{I}-1)}\right\}: \quad=\{\mathbf{0 . 0 2}\}
\end{aligned}
$$

This number gives an idea about the discrepancy between the real structure of the object and the perceived configuration. This example may give a closer idea about our model y compare it with the obtained result from structure in Fig. 10. In this case, both crossing lines intersect the parallel segments with equal angles. It is because that the previous twofold symmetry axis no longer remains. If a $\mathrm{C}_{2}$ rotation is performed to line $\mathrm{L}_{1}$, the new position is the indicated by a segmented line. In any case, because with respect to the parallel lines, this axis remains a $\mathrm{C}_{2}$ axis, this is the symmetry operation we have to apply. With similar number that in the previous case, the results is now: 


$$
\begin{gathered}
\sigma_{1}=\{6 \times 1+9 \times 0.5 ; 6 \times 1+7 \times 0.5 ; 6 \times 1+5 \times 0.5 ; 6 \times 1+3 \times 0.5\} \\
\sigma_{2}=\{6 \times 1+3 \times 0.5 ; 6 \times 1+5 \times 0.5 ; 6 \times 1+7 \times 0.5 ; 6 \times 1+9 \times 0.5\} \\
\sigma_{\mathrm{T}}: \sigma_{1}+C_{2} \sigma_{2}=\{10.5+7.5 ; 9.5+8.5 ; 8.5+9.5 ; 7.5+10.5\}= \\
=\{18 ; 18 ; 18 ; 18\} \\
\pi_{\mathrm{T}}=\left(\wp_{\mathrm{T}} / \sigma_{\mathrm{T}}\right): \quad=\{0.67 ; 0.67 ; 0.67 ; 0.67\} \\
\left\{\pi_{\mathrm{T} 1}\right\}=\left\{\pi_{\mathrm{T} 1}-\pi_{\mathrm{T}(\mathrm{i}-1)}\right\}: \quad=\{\mathbf{0 . 0} ; \mathbf{0 . 0} ; \mathbf{0 . 0}\}
\end{gathered}
$$

This result indicates that the feeling obtained by the observer is parallelism. Lines crossing with similar angle do not change the impression "seen".

From above facts, it is clear that it is possible to extract some type of objective information about the subjective impression got by any observer. The method may be easily generalized.

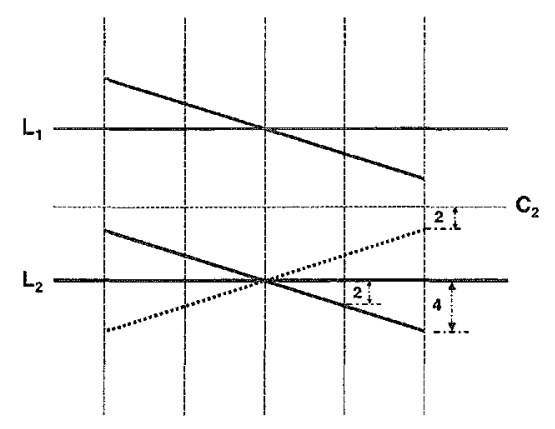

Fig. 10.- Symmetry operations in the case of equal angle crossing lines.

\section{PROPOSAL FOR A SYSTEM TO ANALYZE THE ZÖLLNER ILLUSION}

The model proposed in previous paragraphs needs now some type of implementation in order to get the possibility to be employed in conventional analysis instruments. These instruments, as it is clear, have a different group of applications than usual pattern or image processors. In this case, these systems have interest in fields, such as architecture, painting o even textile design, where the main objective is the impression given by a certain image to an observer and perceived by him. The approach adopted by us will be based on a similar architecture to the existing in the first levels of the visual cortex. Although many other approaches could be possible we have opted by a model with a physiological basis. The reason is that if something like a subjective impression is wished to be obtained, configurations similar to the existing in living bodies may give a behavior similar to the perceived. The general structure to be employed is the one shown in Fig. 11.

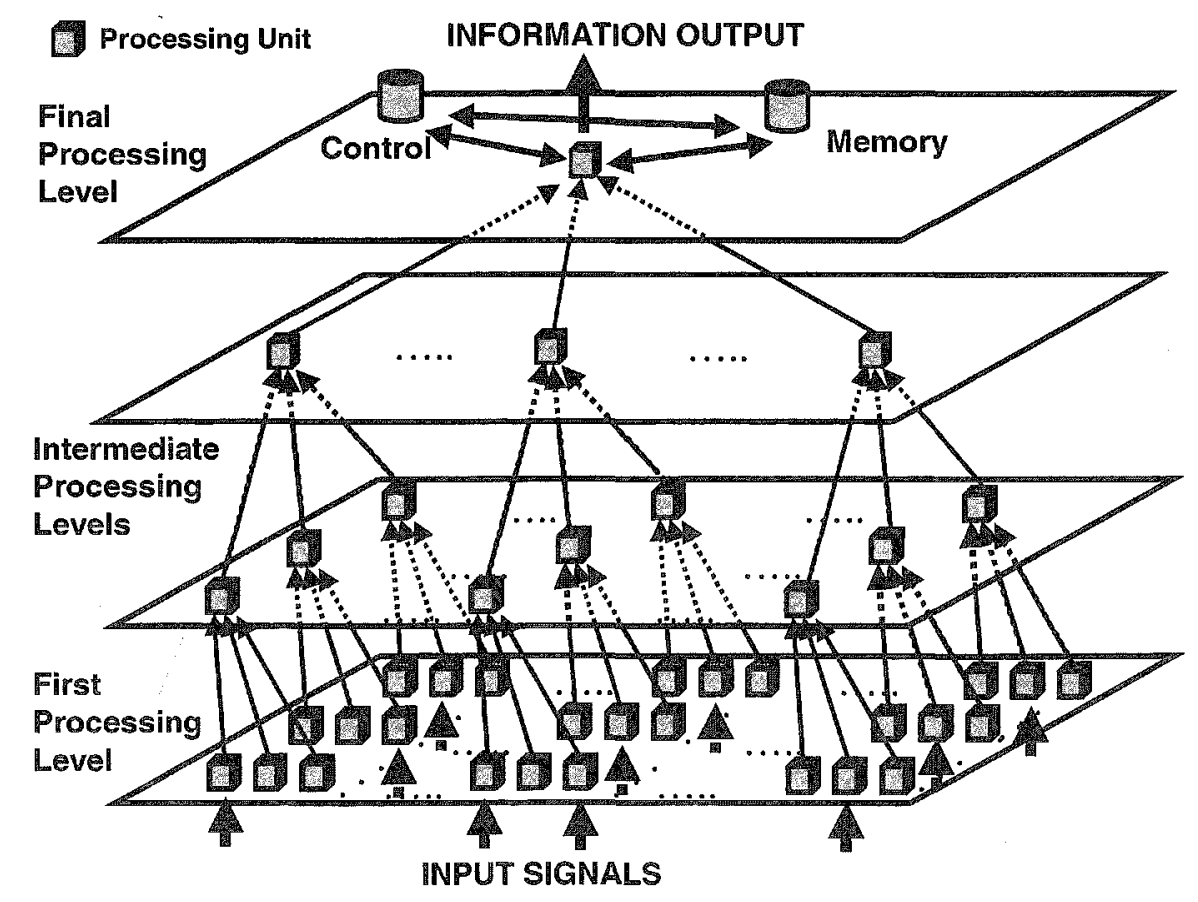

Fig. 11.- Processing levels in the adopted configuration. 


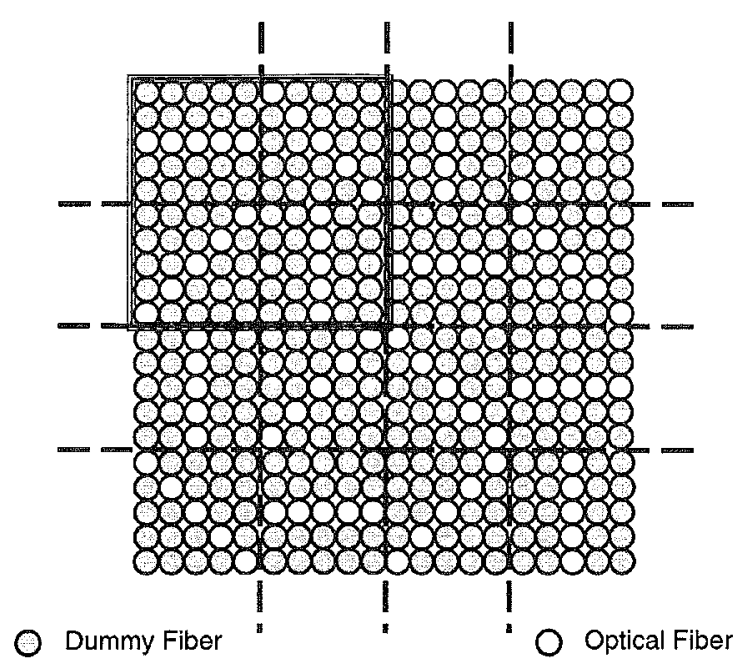

Fig. 12.- Arrangement corresponding to a small part of the sensing area.
Our configuration is based on a certain number of processing levels going from a first one, where main characteristics of image are extracted, to a higher level where processing units are located as well as the final sensing unit from where the resulting information goes out to the decision centre. In between, several other layers perform intermediate tasks needed to the total image processing. Every level has different characteristics and behaviors needing to be studied independently.

The first level is composed by a set of reception units arranged in a way similar to the one shown in Fig. 12. This distribution corresponds to the visual cortex structure indicated in Fig. 7. Different sensing units differ in their possibility to detect different light orientations. In the case of the structure shown in Fig. 12, and in order to get a clearer picture, only four different orientations have been indicated. It is composed by two rows and two columns having in each one of them the four possible orientations adopted in this work. Basic unit is indicated by three line square. The information from an elemental image area impinges over this matrix. If the orientation of the image corresponds with any one of the four possible existing directions in the matrix, a certain signal will appear from it. This signal will depend on the length of the line in the figure. If it covers the whole image, the resulting signal will be four times larger than if only cover one fourth of it. Only $45^{\circ}$ rotations have been represented in Fig. 12, but smaller angles are possible in order to structure a plane similar to the existing in the visual cortex. In the case that more than one orientation appears in the image, corresponding directions are excited. It is obvious that resolution of the matrix and dimensions of the object are quantities directly related. If object dimension is larger than the sensing unit, the four elements will get light and output signal will be the corresponding to these four elements. It is because that, this first level gives coarse information about image characteristics. Processing of finer details is performed at higher levels. Moreover, only different orientations will only be covered by one of these sensing units if image dimension is small enough to be picking up by them. As a matter of fact, it may be proved that the optimum situation corresponds with an elemental image dimension equal to

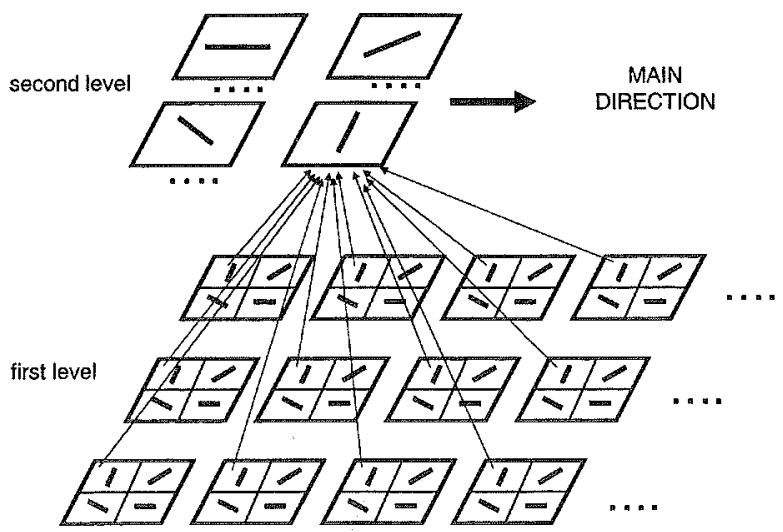

Fig. 13. $=$ Details of connections between first level and second level. the area of one of the small sensing orientation units. These aspects will be analyzed later. The main aspect analyzed in this level is the preferred orientation. If there is not preferred orientation, because signal covers the whole area, the four possible directions will be excited and hence, the output will correspond to the total of the four elements in the matrix. If there is no image, the output will be zero. A first analysis of these signals is performed in the following level. Fig. 14 shows three other elemental units disposed in an enlarged area.

The second level collects information from previous layer. In Fig.13 a part of this recollection is shown. Signals corresponding to four adjacent sensing units in a row, with the same orientation, goes to a processing cell. In the same way, signals from four adjacent units in a column - not shown in figure should go to another processing cell in the same level. Moreover, two more recollections should be performed. They corresponds, in this particular case, to the two other orientations existing in the sensing units, namely, those with $45^{\circ}$ and $135^{\circ}$. The information obtained from this level is which is the main orientation of objects in the image. Sense unit with largest signal will correspond with preferred orientation. In this way, the second layer gives information about the type of signal impinging on the screen and how is oriented. 
The next information needed is related with dimensions and characteristics of the object. To get these details is necessary to obtain some information about how the motives in the image are distributed. This means length of segments and relative distribution. These data are extracted in a similar way to the main direction obtained in second layer. As a matter of fact, they could be obtained in the same second level but for simplicity we have carried it out to a third level. In this case, sensing units with the same orientation than object main direction send information about the signal they get to a register. Each register gets information from each row in the input plane. In this way, information about the length of the line is obtained and, at the same time, distance between existing lines. A similar set of register get information about other directions in the input plane. A simplified detail about this information recollection is shown in Fig. 14. In this case, information about parallel lines in the frontal plane direction is obtained. These information is related with the data employed in the mathematical example indicated in a previous paragraph. This information will be carried out to the upper level where will be processed.

The general scheme of the systems, as a synthesis of the whole process, is shown in Fig. 15. Main blocks are detailed and flux between them indicated. This scheme gives the principal operations and internal details need further explanation. The complexity of these details will be a function of the complexity of image and details to be processed.

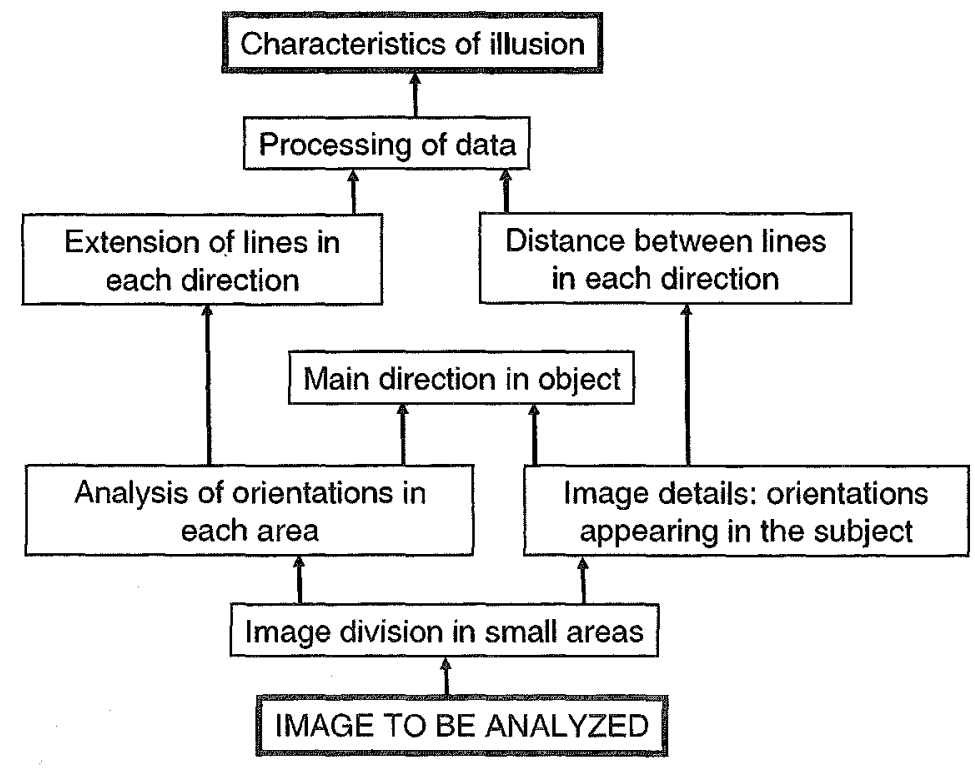

Fig. 15.- General structure of the configuration to analyze parallelism between lines. 


\section{ANALYSIS OF THE WUNDT ILLUSION}

The last effect to be presented in this paper is the Wundt illusion. As we have seen in previous paragraphs, depending on the relative position of two straight lines, one in a vertical position and horizontal the other one, we perceive different length although both have the same length. In order to explain this effect we have introduced the concept of the "internal subjective vanishing point", explained before, and the corresponding horizon line. Although this illusion will not be studied in the same way than the preceding effect, we are going to offer some general ideas that will be developed in a future paper.

When an observer looks to lines in Fig. 2 he does not analyze them in an individual way. The first thing he observes is their relative position. He internally "creates" a horizon line and with reference to it locates the lines. The line at a lower position, either the horizontal line or the lowest part of the vertical one, determines the point closest to the observer. Distances and lengths are now taken with relation to the "distance" between that lower position and the vanishing point. Because this case is very simple, there is not any need to go to a two-point perspective or, in general, to a multi-point perspective, as it is common in many artists. A very straightforward method may be applied now to determine some type of length "seen" by an observer as it was done in the Zöllner illusion. Details about that will be reported in an other place.

\section{CONCLUSIONS}

The Zöllner illusion has been studied and a simple model to justify some of its characteristics has been reported. Moreover, a first approach to an artificial system able to work in a similar way to living bodies have been presented. This work is just a part of a more extended method to analyze visual illusions. Wundt illusion has been initiated in this paper too and Müller-Lyer was studied by us in a previous paper. The employed methods have in the three cases some common points but there is yet a need to integrate them in a more general theory. The cases presented in Fig. 5 need the introduction of some new concepts. For example, the influence of the concept that we have called "subjective weight" or "visual density" has not been employed in the present paper. But the proof that it is possible to "put numbers to visual illusions" has been clarified in this work. A final point should be indicated. In this paper a certain global approach to study visual illusions has been presented. But some more practical aspects, related with the way visual signals are processed and related, have to be introduced. This facts have to related with the form signals go from retina to visual cortex. If a system with similar properties to some visual systems in living bodies is to be developed, similar type of signals should be employed. This approach has been reported by us in previous papers ${ }^{11-12}$ and should be connected with the present one.

\section{ACKNOWLEDGMENTS}

This work was partly supported by CICYT "Comisión Interministerial de Ciencia y Tecnología", grant TIC99-1131 and CAM "Comunidad Autónoma de Madrid", grant 07T/0037/2000.

\section{REFERENCES}

1. R. L. Gregory, "Eye and Brain. The Psychology of Seeing". Oxford University Press. Oxford. 1990.

2. Stephen E. Palmer, "Vision Science. Photons to Phenomenology". The MIT Press. Cambridge, Mass. 1999.

3. M. Luckiesh, "Visual Illusions. Their Causes, Characteristics \& Applications". Dover. New York 1965.

4. R. L. Solso, "Cognition and the Visual Arts". The MIT Press. Cambridge, Mass. 1994.

5. "Visual Cognition", Eds.: S. M. Kosslyn and D. N. Osherson, The MTT Press. Cambridge, Mass.1995.

6. J.A. Martin-Pereda and A. Gonzalez-Marcos, "A new method to analyse the perception of size". Applications of Digital Image Processing XXIV. SPIE. Vol 4472 pp. 75-84 (2001)

7. D.H. Hubel and T.N. Wiesel, "The Ferrier Lecture: Functional architecture of macaque monkey visual cortex", Proc. $R$. Soc. London. B 198, 1-59 (1977).

8. G.M. Shepherd, "The Synaptic Organization of the Brain". Oxford. 1990.

9. "Neuro-vision Systems. Principles and Applications". Eds.: Madam M. Gupta and George K. Knopf. IEEE Press. New York. 1993.

10. J.G. Nicholls, A. Robert M. and B.G. Wallace, "From Neuron to Brain", Sinauer Associates, Inc. Sunderland, MA. 1992. 
11. J.A. Martín-Pereda and A. González-Marcos, "Image processing based on a model of the mammalian retina". Applications of Photonics Technology 3. Closing the Gap between Theory, Development, and Applicaction. Edited by G.A.Lampropoulos and R.A. Lessard. SPIE, vol. 3491, 1185-1190, (1998).

12. Jose A. Martin-Pereda \& Ana P. Gonzalez-Marcos, "Image characterisation based on the mammalian visual cortex". Applications of digital Image Processing XXII, SPIE, vol. 3808, pp. 614-623, (1999). 